\title{
Automatic Detection of Open and Closed Separation and Attachment Lines
}

\author{
David N. Kenwright ${ }^{*}$ \\ MRJ Technology Solutions, NASA Ames Research Center
}

\begin{abstract}
A fully automatic feature detection algorithm is presented that locates, and distinguishes, lines of flow separation and attachment on surfaces in 3-D numerical flow fields. The algorithm is based on concepts from 2-D phase plane analysis of linear vector fields. Unlike prior visualization techniques based on particle tracing or flow topology, the phase plane algorithm detects separation using local analytic tests. Results show that it not only detects the standard closed separation lines but also the illusive open separation lines which are not captured by flow topology methods.
\end{abstract}

\section{INTRODUCTION}

Flow separation and attachment occur when a flow abruptly moves away from or returns to a solid body such as the surface of an aircraft. The lines along which this occurs are called separation and attachment lines. Flow separation is most prevalent when aircraft fly at slow speeds and high angles of attack. A separated airflow causes a significant increase in drag and raises the stall speed of an aircraft. This is particularly dangerous at takeoff and landing because it can cause an aircraft to stall and rapidly lose altitude. The ability to predict when and where flow separation occurs is clearly very important to aircraft designers.

Several techniques have been used to identify separation and attachment lines in numerical flow data sets although these generally fall into one of two categories: user observation or feature detection. Those based on user observation require scientists to study the flow patterns on a surface and use their experience or insight to identify the relevant features. This approach is used in all experimental studies and in most numerical studies of separation. In contrast, techniques based on feature detection can automatically locate the position of separation or attachment lines with little or no human intervention.

Feature detection techniques are becoming increasingly important for analyzing large data sets (i.e., 1-1000 Gigabytes) which are larger than the memory capacity of many graphics workstations. A major advantage of feature detection algorithms is that they can be executed on computers without graphics capability, such as the supercomputers that generate the data. These algorithms output 3-D graphics primitives whose combined size is typically three orders of magnitude smaller than the original data set.

"NASA Ames Research Center, Mail Stop T27A-2,

Moffett Field, CA 94035, USA.

davidk@nas.nasa.gov
This paper describes an automatic and deterministic feature detection algorithm that locates both separation and attachment lines on grid surfaces in numerical flow data sets. This algorithm is based on phase plane analysis and performs a local analysis of the vector field rather than a global analysis of the entire flow field. This type of algorithm is useful for analyzing large partitioned data sets, such as those computed on distributed memory architectures, because the cells can be processed independently. Also, it lends itself to parallel processing because the algorithm is inherently parallel.

The contents of the paper are organized as follows. Prior work on the detection and visualization of separation and attachment lines is discussed in Section 2. Important findings from experimental and theoretical studies of separated flows are also discussed in that section. The mathematical foundations for the current algorithm and aspects of phase plane analysis are covered in Section 3. Details of the algorithm are presented in Section 4, and applications are shown in Section 5. The results are compared to those produced by particle tracing, line integral convolution, and flow topology algorithms on the same data sets. Issues are discussed in Section 6.

\section{LITERATURE REVIEW}

\subsection{Numerical Approaches}

A common approach for visualizing separation and attachment lines is to place seed particles near a body and to compute integral curves, such as stream or streak lines, which are constrained to the body. This approach can be effective if large numbers of particles are released because the curves merge together along separation lines (see Figure 5(a)). Attachment lines are not usually so obvious because particles diverge from these lines. Because this approach relies on observation, the analyst must interpret the flow patterns to determine which lines correspond to separation and which to attachment. Both are visually similar, although it can usually be determined by examining the direction of the asymptotes in relation to the direction of the onset flow. The asymptotes curve downstream along separation lines and upstream along attachment lines. However, it becomes difficult to make the same distinction when the separation lines are perpendicular to the onset flow.

Skin friction lines are often studied instead of particle traces because they are the numerical analog of surface oil flow techniques used in wind tunnel experiments [1]. Skin friction lines are computed from the body or wall shear stress, which is defined as the normal derivative to the wall of the velocity vector [1]. The shear stress vectors point in the direction of the near-wall velocity 


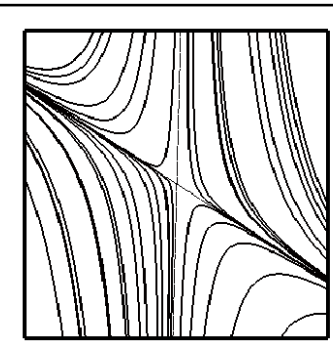

Saddle

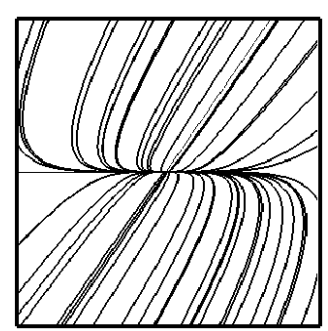

Improper Node

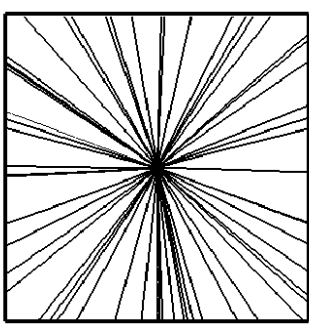

Proper Node

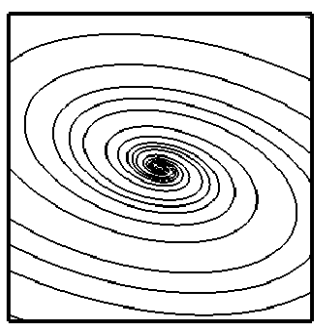

Spiral

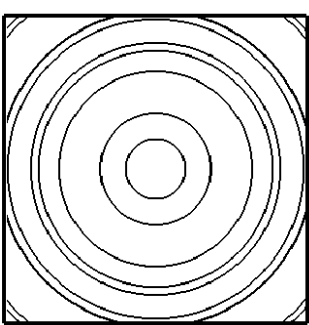

Center

Figure 1. Five phase portraits that arise in linear vector fields.

vectors when projected normal to the wall. The skin friction lines are integral curves of the shear stress vector field. As with particle traces, the analyst must look for converging or diverging patterns in order to locate the separation and attachment lines.

Texture synthesis techniques developed for vector field visualization create continuous flow patterns rather than just discrete lines [2], [3]. These techniques have been applied to CFD data sets to create flow patterns that are strikingly similar to experimental visualizations (see Figure 5(b)). Once again, these techniques rely on user observation of flow patterns. While this is relatively easy to do in steady-state simulations, it becomes difficult in transient simulations when the surface flow patterns change significantly over time. In [2], the traditional gray-scale texture images were colored by a scalar quantity, the angle between the velocity vector and the surface normal. This was based on the observation that the on and off surface flow is high along separation and attachment lines. Although this highlights regions of general interest, it does not specifically locate separation and attachment lines.

Only one feature detection technique has previously been published that can automatically locate separation and attachment lines [4]. It is based on the concepts of vector field topology. The topology of a vector field consists of critical points, i.e., points where the velocity is zero, and tangent curves (instantaneous streamlines) which connect these points. Because the velocity at a critical point is zero, the velocity field in the neighborhood of the critical point is determined by the velocity gradient tensor, $\operatorname{grad}(\boldsymbol{u})$. Critical points are classified, to a first order approximation, by the eigenvalues and eigenvectors of $\operatorname{grad}(\boldsymbol{u})$. Common classifications include a saddle, node, spiral, and center (see Figure 1).

In [4], the separation lines were generated by integrating outwards from the saddle or node type critical points in the real eigenvector directions. These tangent curves, or more precisely, the separatrices, were classified as separation or attachment lines based on the sign of the eigenvalues. That is, a positive or a negative real part of an eigenvalue indicated whether the tangent curve had an attracting or repelling nature. This approach assumes that the separation is closed, that is, a separation line that begins at a saddle or node will end at another saddle or node.

Another type of separation, called open separation, does not require separation lines to either start or end at critical points. Consequently, flow topology does not predict open separation. The theory for open separation also suggests that a separation line can terminate at a critical point without originating from one. An example of this will be shown in Section 5.1. Open separation has been observed by experimentalists in wind and water tunnel experiments, but this phenomenon has not been previously studied by the numerical flow visualization community.

\subsection{Experimental and Topological Approaches}

There has been extensive research on three-dimensional flow separation over the last 50 years. Important contributions have come from Lighthill [5], Tobak and Peak [6], Wang [7], Dallman [8], Zhang [9], Chong, Perry, and Cantwell [10] and [11], and Chapman [12]. These contributions can be categorized as either experimental or topological. The experimental approach is based on observations made in wind and water tunnel experiments, whereas the topological approach is based on the mathematics of Poincare [13].

One of the most important developments was the concept of open separation [7]. The issue of open separation has been, and still is, one of the most controversial aspects of flow separation. Outstanding questions reported in [14] include: What is the nature of the starting point of an open separation line? How does closed separation evolve into open separation? Under what circumstances will open separation occur or not occur? This paper does not attempt to answer such questions. However, it may contribute to the answers because the visualization technique presented here can detect open separation in numerical vector fields. Results presented in Section 5.2 support assertions made by Chapman [12] that open separation is prominent in delta-wing configurations.

The definition of a separation line was another long standing dispute in flow separation literature. Specifically, is a separation line an envelope of the limiting streamlines or the skin friction lines, or is it itself a limiting streamline? Zhang [9] settled this dispute by showing that both definitions were partly correct. That is, a separation line is an envelope if based on boundary layer theory, but it is a limiting streamline if based on the Navier-Stokes equations. The latter definition is used in this paper.

\section{THEORY}

It is assumed that the computational domain on the surface can be subdivided into triangles and the Cartesian vector components are given at the vertices. Based on these data, a continuous linear vector field can be constructed that passes through each triangle and satisfies the prescribed vectors at the vertices: 


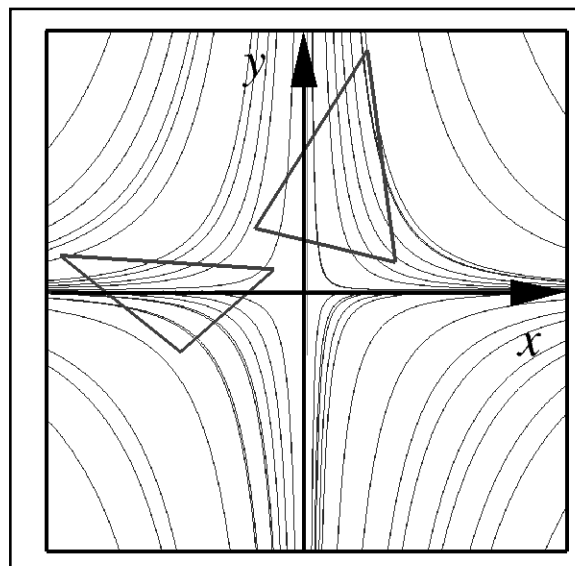

(a) Saddle: $\mu<0<\lambda$

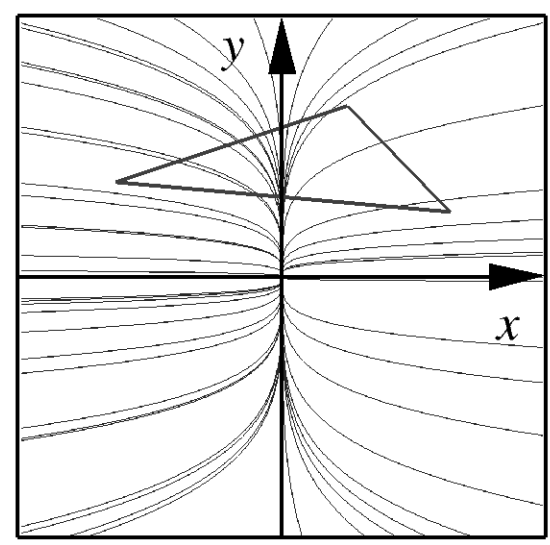

(b) Repelling node: $0<\mu<\lambda$

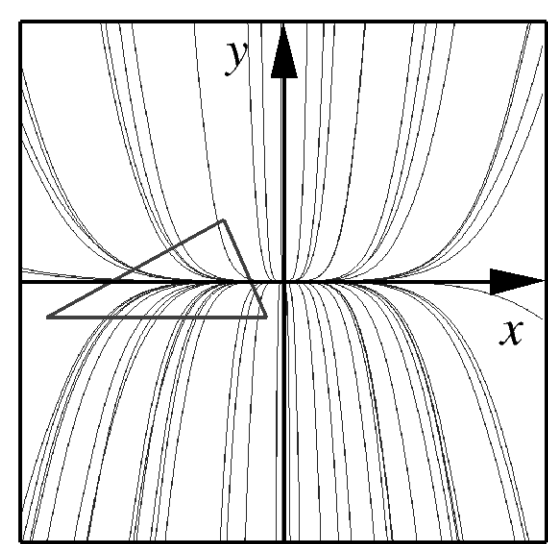

(c) Attracting node: $\mu<\lambda<0$

Figure 2. Three phase portraits have tangent curves that asymptotically converge on an axis in the phase plane. Triangles that straddle these axes contribute line segments to either separation or attachment lines.

$$
\left(\begin{array}{l}
\dot{\mathrm{x}} \\
\dot{\mathrm{y}}
\end{array}\right)=\left(\begin{array}{l}
\mathrm{a}_{1} \\
\mathrm{a}_{2}
\end{array}\right)+\left(\begin{array}{ll}
\mathrm{b}_{1} & \mathrm{c}_{1} \\
\mathrm{~b}_{2} & \mathrm{c}_{2}
\end{array}\right)\left(\begin{array}{l}
\mathrm{x} \\
\mathrm{y}
\end{array}\right)
$$

Here, $x$ and $y$ are the Cartesian coordinates, and $(\dot{x}, \dot{y})$ is the tangential velocity or shear stress vector. The coefficients $\left(a_{1}, a_{2}\right)$ and those in the $2 \times 2$ (Jacobian) matrix on the right-hand side of equation (1) are constants. These constants can be computed analytically by substituting the coordinates and vectors from each vertex into equation (1) and then solving the resulting set of simultaneous equations:

$$
\left(\begin{array}{l}
\mathrm{a}_{1} \\
\mathrm{~b}_{1} \\
\mathrm{c}_{1}
\end{array}\right)=\left(\begin{array}{lll}
1 & \mathrm{x}_{1} & \mathrm{y}_{1} \\
1 & \mathrm{x}_{2} & \mathrm{y}_{2} \\
1 & \mathrm{x}_{3} & \mathrm{y}_{3}
\end{array}\right)^{-1}\left(\begin{array}{l}
\dot{\mathrm{x}}_{1} \\
\dot{\mathrm{x}}_{2} \\
\dot{\mathrm{x}}_{3}
\end{array}\right)
$$

and

$$
\left(\begin{array}{l}
\mathrm{a}_{2} \\
\mathrm{~b}_{2} \\
\mathrm{c}_{2}
\end{array}\right)=\left(\begin{array}{lll}
1 & \mathrm{x}_{1} & \mathrm{y}_{1} \\
1 & \mathrm{x}_{2} & \mathrm{y}_{2} \\
1 & \mathrm{x}_{3} & \mathrm{y}_{3}
\end{array}\right)^{-1}\left(\begin{array}{l}
\dot{y}_{1} \\
\dot{y}_{2} \\
\dot{y}_{3}
\end{array}\right)
$$

By differentiating equation (1) with respect to time and then algebraically manipulating the resulting equations, it can be separated into a pair of second order non-homogeneous ordinary differential equations:

$$
\begin{aligned}
& \ddot{x}-\left(b_{1}+c_{2}\right) \dot{x}+\left(b_{1} c_{2}-b_{2} c_{1}\right) x=\left(a_{2} c_{1}-a_{1} c_{2}\right) \\
& \ddot{y}-\left(b_{1}+c_{2}\right) \dot{y}+\left(b_{1} c_{2}-b_{2} c_{1}\right) y=\left(a_{1} b_{2}-a_{2} b_{1}\right)
\end{aligned}
$$

The solutions to these types of equations can be found in most texts on differential equations (e.g., [15], [16]). The eigenvalues of this system are the roots of the homogeneous part of equation (3), i.e., $\lambda^{2}-\left(b_{1}+c_{2}\right) \lambda+\left(b_{1} c_{2}-b_{2} c_{1}\right)=0$. If the determinant of the Jacobian matrix is non-zero, the solution takes the form:

$$
\left(\begin{array}{l}
\mathrm{x}(\mathrm{t})-\mathrm{x}_{\mathrm{cp}} \\
\mathrm{y}(\mathrm{t})-\mathrm{y}_{\mathrm{cp}}
\end{array}\right)=\left(\begin{array}{ll}
\xi_{1} & \eta_{1} \\
\xi_{2} & \eta_{2}
\end{array}\right)\left(\begin{array}{l}
\alpha e^{\lambda t} \\
\beta e^{\mu t}
\end{array}\right)
$$

where $\lambda$ and $\mu$ are the eigenvalues of the Jacobian matrix and $\left(\xi_{1}, \xi_{2}\right)^{T}$ and $\left(\eta_{1}, \eta_{2}\right)^{T}$ are the eigenvectors. The two column eigenvectors form the eigenmatrix. It is important that the eigenvectors are scaled so that the determinant of the eigenmatrix equals 1 . The reason for this will become apparent. The terms $\alpha$ and $\beta$ are arbitrary constants that define a particular curve in the phase plane. The constants $x_{c p}$ and $y_{c p}$ are the coordinates of the critical point:

$$
\mathrm{x}_{\mathrm{cp}}=\frac{\mathrm{a}_{2} \mathrm{c}_{1}-\mathrm{a}_{1} \mathrm{c}_{2}}{\mathrm{~b}_{1} \mathrm{c}_{2}-\mathrm{b}_{2} \mathrm{c}_{1}} \quad \mathrm{y}_{\mathrm{cp}}=\frac{\mathrm{a}_{1} \mathrm{~b}_{2}-\mathrm{a}_{2} \mathrm{~b}_{1}}{\mathrm{~b}_{1} \mathrm{c}_{2}-\mathrm{b}_{2} \mathrm{c}_{1}}
$$

In equation (4), these constants translate the coordinate system so that the origin coincides with the critical point. Note that every triangle has a critical point somewhere in its linear vector field, although, in general, the critical point will not lie inside the triangle. The critical points that do lie inside triangles correspond to those found by vector field topology methods [4]. Equation (4) can be simplified further by changing to a canonical coordinate system, that is, a coordinate system where the eigenvector directions are orthogonal:

$$
\left(\begin{array}{l}
x \\
y
\end{array}\right)=\left(\begin{array}{ll}
\xi_{1} & \eta_{1} \\
\xi_{2} & \eta_{2}
\end{array}\right)^{-1}\left(\begin{array}{l}
x \\
y
\end{array}\right)=\left(\begin{array}{l}
\alpha e^{\lambda t} \\
\beta e^{\mu t}
\end{array}\right)
$$

The lines of the vector field can now be represented by curves in the $(x, y)$ plane (often referred to as the Poincare phase plane [13]). Note that italic characters denote coordinates or vectors that have been mapped into the phase plane. By eliminating the integration variable $t$ from equation (6), the trajectories of these curves can be expressed as an implicit scalar function. If the eigenvalues are both real numbers, the expression is either: 


$$
\Psi(x, y)=\frac{x^{\mu}}{y^{\lambda}} \quad \text { or } \quad \Psi(x, y)=-\frac{y^{\lambda}}{x^{\mu}}
$$

Both scalar functions are valid solutions to equation (1). The contours of $\Psi(x, y)$ are everywhere tangent to the vector field and may be verified using the relationship $\nabla \Psi \bullet \boldsymbol{u}=0$, where $\boldsymbol{u}$ is the image of the vector field in the phase plane. By differentiating equation (6) with respect to t, the reader can obtain the necessary transformation that maps the vector field (equation (1)) into the phase plane. It now becomes apparent why the determinant of the eigenmatrix must equal one; it ensures that the vector field is not scaled by the canonical transformation.

By choosing arbitrary points in the phase plane and rendering tangent curves using equation (7), we obtain the so-called phase portrait of the system. There are five different phase portraits for the linear vector field described by equation (1), as shown in Figure 1. Equation (7) gives rise to the phase portraits for the saddle and the improper and proper nodes. The saddle arises when the eigenvalues have opposite signs, whereas the nodes arise when they have the same sign.

One definition of a separation line, as discussed in Section 2.2, is a limiting streamline on which adjacent streamlines converge. The phase portrait for the saddle (Figure 2(a)) contains two lines on which streamlines converge. These lines originate at the critical point and are tangential to the eigenvector directions, i.e., the $x=0$ and $y=0$ axes in the phase plane. These lines are called separatrices in phase plane terminology. By substituting either $x=0$ or $y=0$ into equation (7), we find that the stream function is either zero or singular depending on which solution is used. In either case, these lines correspond to streamlines and fulfill the definition of a separation line.

The phase portrait for the improper node can assume one of two orientations in the phase plane depending on whether it is an attracting node $(\mu<\lambda<0)$ or a repelling node $(0<\mu<\lambda)$. Specifically, streamlines asymptotically converge on the $x=0$ axis for a repelling node, while they converge on the $y=0$ axis for an attracting node (Figures 2(b) and 2(c)). A node also has a degenerate form called a proper node (see Figure 1) which arises when one of the eigenvalues is zero. The phase portrait for a proper node does not have any limiting streamlines and does not therefore contribute to separation or attachment lines.

The phase portraits for the spiral and center, also shown in Figure 1, arise when the eigenvalues are complex conjugates, i.e., $\mu=\sigma+i \nu, \lambda=\sigma-i \nu$. Equation (7) still holds for these cases, although it can be re-derived in terms of real numbers by first converting to polar coordinates and then by applying standard trigonometric identities. The expression for $\Psi$ now becomes:

$$
\Psi(r, \theta)=r e^{\frac{\sigma}{v} \theta}
$$

where $r=\sqrt{x^{2}+y^{2}}$ and $\theta=\tan ^{-1} y / x$. Complex eigenvalues are indicative of rotating flows and typically occur near vortices. Note that the center is just a degenerate form of the spiral where the eigenvalues are pure imaginary numbers, i.e., $\sigma=0$. The phase portraits for the spiral and the center do not exhibit any limiting lines like those for the saddle and improper node. This implies that there are no separation lines in vortical flow regions according to the definition used here. Consequently, separation lines will terminate as they enter vortices in the present algorithm.

$\Psi(x, y)$ and $\Psi(r, \theta)$ behave much like Lagrange's stream function for irrotational, divergence free, 2-D vector fields inasmuch as the tangent lines are contours of a scalar function. However, $\Psi(x, y)$ and $\Psi(r, \theta)$ are exact solutions to a rotational 2-D linear vector field which, in general, will not be divergence free. No prior references have been found for these scalar functions in texts on differential equations. The author calls them nonconservative stream functions because they violate the law of mass conservation. It can be shown that the divergence of equation (1) is non-zero by calculating the trace of the Jacobian:

$$
\nabla \bullet \mathbf{u}=\mathrm{b}_{1}+\mathrm{c}_{2} \neq 0
$$

where $\mathbf{u}=(\dot{\mathrm{x}}, \dot{\mathrm{y}})$. A non-zero divergence means that mass is not conserved on the surface of the triangle. The fact that this system can 'lose mass' is physically important because this accounts for fluid that leaves the surface as the flow converges on a separation line. Conversely, the system will 'gain mass' as the flow returns to the surface and diverges from an attachment line.

\section{IMPLEMENTATION}

The following algorithm describes how to detect a separation or attachment line on a triangular element from the vectors prescribed at the vertices. In general, these vectors will not lie in the plane of the triangle and must be projected onto the surface by calculating either the tangential velocity vectors or the shear stress vectors. This projection is relatively simple for curvilinear grids if they are transformed into an orthogonal coordinate system (computational coordinates) since one coordinate direction is always orthogonal to a no-slip surface. For unstructured meshes, the velocity vectors can be projected based on a local surface normal. This projection should be done before executing the following steps.

1. Transform the triangle's coordinates and vectors from a three-dimensional basis into a two-dimensional basis. These will be referred to as 2- $D$ coordinates herein.

2. Calculate the coefficients of the 2-D linear interpolation function using equation (2) and assemble the Jacobian matrix shown in equation (1).

3. Calculate the discriminant of the Jacobian matrix using $\Delta=t r^{2}-4($ det $)$, where $t r$ is the trace of the Jacobian $\left(\mathrm{b}_{1}+\mathrm{c}_{2}\right)$ and det is the determinant $\left(\mathrm{b}_{1} \mathrm{c}_{2}-\mathrm{b}_{2} \mathrm{c}_{1}\right)$. Processing stops if the discriminant is negative, that is, when the eigenvalues are complex numbers.

4. Evaluate the eigenvalues of the Jacobian matrix. Processing stops if one of the eigenvalues is zero (i.e., the phase portrait is a proper node).

5. Calculate the eigenvectors of the Jacobian matrix and assemble the eigenmatrix as shown in equation (4).

6. Calculate the coordinates of the critical point using equation (5).

7. Project the triangle onto the phase plane by transforming each vertex into canonical coordinates using equation (6).

8. Determine the phase portrait using the eigenvalue tests in 
Figure 2. The phase portrait will be either a saddle or a repelling/attracting improper node at this stage.

9. If the phase portrait is a saddle or a repelling node, determine whether the line $\boldsymbol{x}=\mathbf{0}$ intersects the triangle on the phase plane. If it does, calculate the two intercepts and convert them back into 2-D coordinates using the inverse transformation of step 7. The line segment connecting these points will form part of an attachment line.

10. If the phase portrait is a saddle or an attracting node, determine whether the line $\boldsymbol{y}=\mathbf{0}$ intersects the triangle on the phase plane. If it does, calculate the two intercepts and convert them back into 2-D coordinates using the inverse transformation of step 7. The line segment connecting these points will form part of a separation line.

11. Transform the 2-D coordinates back into 3-D Cartesian coordinates and render the line segment with a color that distinguishes it as a separation or attachment line.

These steps are independently applied to every triangle on the surface of the body and may be executed in serial or in parallel. A C++ implementation of this algorithm, running on a Silicon Graphics Onyx II with one R10000 CPU, processed approximately $10^{5}$ triangles per second.

\section{RESULTS}

Many numerical and experimental studies of separated flows have concentrated on bodies with simple geometries. Both data sets considered here fall into this category. The first is a hemisphere (or ogive) cylinder which is representative of the forebody of an aircraft. The second is a $65^{\circ}$ swept delta wing which is representative of the wing shape used on many fighter aircraft. In spite of their simple geometries, these data sets display complicated separation patterns and contain both open and closed separation and attachment lines.

\subsection{Hemisphere Cylinder}

The hemisphere cylinder in Figure 3(a) has been used in both experimental and numerical studies of flow separation over an inclined body [17]. The surface flow pattern shown in Figures 3(a) and 3(b) was computed using a line integral convolution (LIC) program [2]. Separation and attachment lines are clearly visible where the streaks converge. However, the LIC algorithm does not distinguish them or find their location.

The flow topology of the hemisphere cylinder was previously examined by Helman and Hesselink [4] using numerical techniques based on critical point theory. The surface topology shown in Figure 3(a) was produced using the topology module in FAST [18] and is consistent with the results presented by Helman and Hesselink. The tangential velocity field on the no-slip body (i.e., the grid plane $\mathrm{k}=0$ ) was generated by projecting the velocity vectors in the grid plane next to the surface $(\mathrm{k}=1)$ onto the body. The local phase plane algorithm was applied to the same tangential velocity field and produced the results shown in Figure 3(b). The separation lines are white and the attachment lines are black in both images.

There are two obvious differences between Figures 3(a) and 3 (b). The first is that the separation and attachment lines produced by the phase plane approach do not connect all the critical points. This is because many of the lines connecting the critical points are not separation or attachment lines according to the definition used here. In particular, if the reader examines the flow around the hemisphere at the front of the body in Figure 3(b), there are no asymptotically converging flow patterns in this region. The second obvious difference is that the topology algorithm in FAST does not detect two attachment lines on the rear of the body. The same lines were also missing in the results presented in [4]. The surface flow pattern in Figure 3(b) clearly shows that the flow converges along these lines. These are open attachment lines according to Chapman [12] because they terminate at a critical point but do not originate from one. Presumably, the topology approach could detect these lines if the integration time step were reversed and the streamlines traced backwards from the terminating critical points.

\subsection{Delta Wing}

Many of today's fighter aircraft are based on delta wing geometries like the one shown in Figure 4 and 5. Aeronautical engineers are particularly interested in the behavior of the flow both on and above the wing while flying at low speeds and high angles of attack [19]. Figure 5(a) shows the surface streamlines computed from the tangential velocity field. Note that there are many asymptotically converging streamline patterns running parallel to the lead edges of the wing. Chaderjian and Schiff [19] used traces like these to analyze this data set and reported the following. "The technique clearly reveals the separation lines where particles accumulate. Reattachment lines are not as readily apparent, since on attachment lines the particles move away from each other. However, computed primary, secondary and tertiary separation lines are readily seen."

The separation lines Chaderjian and Schiff mention are linked to the vortical flow above the wing. This flow was revealed by rendering vector arrows on a transverse plane, as shown in Figure 4(b). The primary and secondary vortices are clearly visible in this figure, but the tertiary vortex, which lies in between them, is less obvious because it hugs the surface. Each vortex draws fluid off the surface along separation lines and returns fluid to the surface along attachment lines. Consequently, the latter are often called re-attachment lines. Given that each vortex is both drawing fluid and returning it to the surface, we expect to see an equal number of separation and attachment lines.

Figure 5(b) shows the surface flow pattern produced by a LIC algorithm. The continuous texture improves on the discrete streamlines in Figure 5(a), although the flow patterns still require careful observation to distinguish the separation lines from attachment lines.

Figure 5(c) shows the critical points and surface flow topology computed using the FAST [18] topology module. Surprisingly, only two pairs of critical points were found on the surface of the wing. A more comprehensive critical point analysis of the entire 3-D flow field revealed that there were no off-surface critical points whatsoever in this data set. Each pair of critical points consists of one repelling spiral point and one saddle point. Note that only one of the integral curves that originates from each saddle point follows an attachment line. None of the other primary, secondary, or tertiary separation or attachment lines near the wing's leading edge either start or end at critical points. These are open separation/attachment lines according to Wang's classification [7], [14]. Flow topology methods cannot detect 

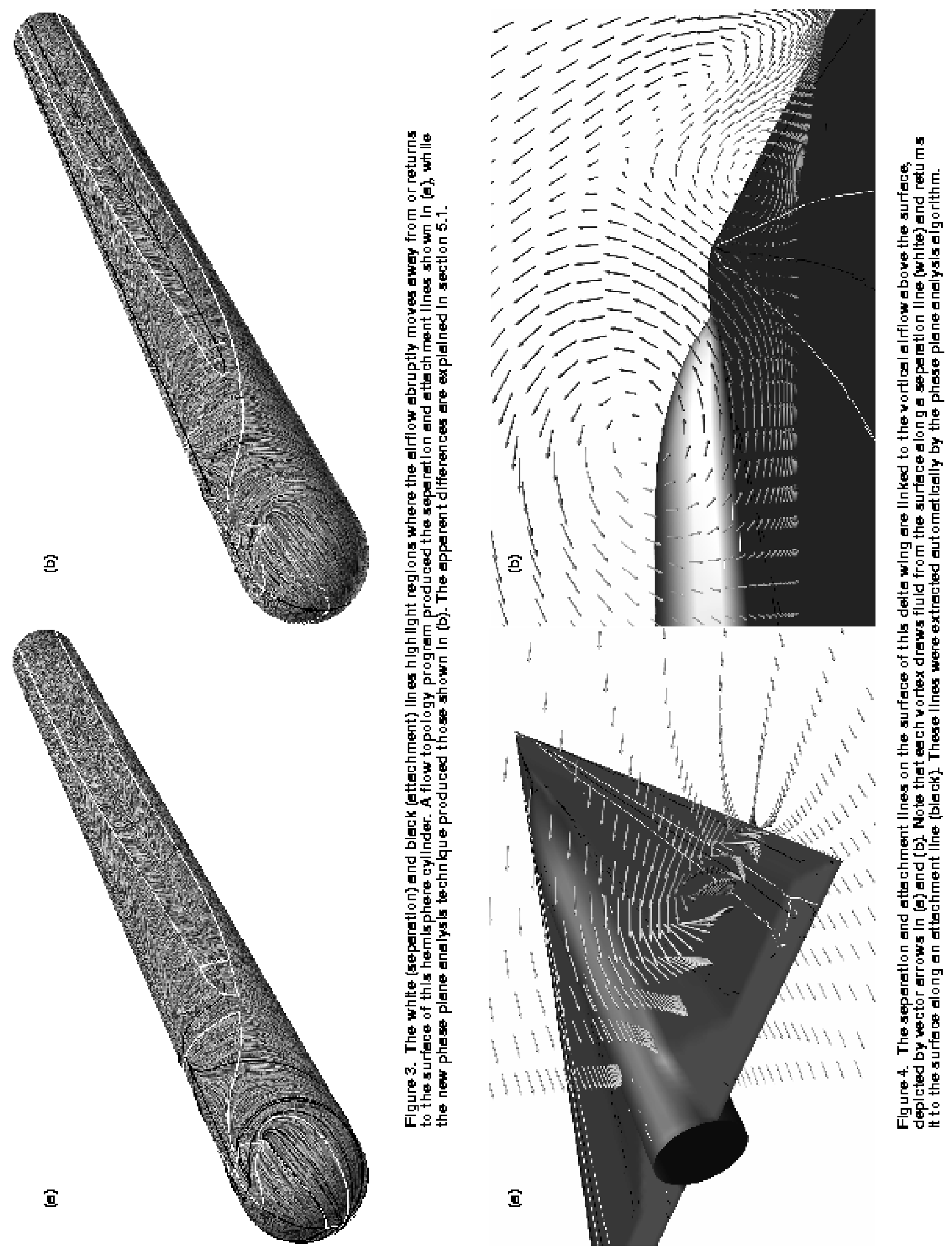
this type of open separation line because they are not bounded by any critical points, either on or off the body.

Figure 5(d) shows the results of the phase plane analysis technique. Separation lines are colored white and attachment lines green. As expected, there are three separation lines and three attachment lines on each side of the wing, that is, one pair for each vortex. Furthermore, their location precisely coincides with the asymptotes of the streamlines. Note the appearance of the disjointed line segments towards the rear of the wing where the separation lines are bent by a vortex. This is a consequence of the low-order linear interpolation function on which the algorithm is based. The location and direction of the separation line are dictated by the gradients of the interpolation function, which are constant over each triangle but discontinuous between triangles. This discontinuity becomes apparent when separation lines curve. Fortunately, most separation lines do not have significant curvature, and the current approach produces acceptable results.

\section{ISSUES}

One problem with the phase plane approach already discussed was the appearance of disjointed line segments. A related problem occurs when flow separation/attachment is relatively weak and becomes diffused over several cells. This causes the phase plane algorithm to either detect multiple "ghost" lines or leave gaps. The attachment line near the center of the wing in Figure 5(d) displays these characteristics. In this event, the assumption that the flow is locally linear is not entirely adequate. Although these lines are visually distracting, they are far less important from an engineering perspective than the strong separation and attachment lines that are well defined. Nevertheless, the author plans to correct this problem by applying the same principles to higher-order interpolation functions.

\section{CONCLUSIONS}

An analytic technique for detecting separation and attachment lines was presented which only requires a local analysis of a surface flow vector field. The theory for the new technique, based on concepts from linear phase plane analysis, was shown to satisfy an accepted definition for a separation line based on the Navier-Stokes equations. The phase plane algorithm was applied to a hemispherical cylinder and a delta-wing data set used in prior numerical studies of separated flows. It detected both open and closed type separation and attachment lines in both data sets. The ability to detect open separation lines is particularly significant because this type of separation is not predicted by flow topology theory, although it has been observed in wind tunnel experiments.

\section{ACKNOWLEDGMENTS}

This work was supported by NASA contract NAS2-14303. The author thanks Susan Ying for the hemisphere cylinder data set and Neal Chaderjian for the delta wing data set. Special thanks to Randy Kaemmerer for his meticulous proofreading, to Vee Hirsch for the video production, and to Creon Levit and Chris Henze for many interesting discussions on flow separation.

\section{REFERENCES}

[1] Pagendarm, H.-G., Walter, B., Feature Detection from Vector
Quantities in a Numerically Simulated Hypersonic Flow Field in Combination with Experimental Flow Visualization, in Proceedings of Visualization '94, IEEE Computer Society Press, 1994, pp.117-123.

[2] Okada, A., and Kao, D., Enhanced Line Integral Convolution with Flow Feature Detection, in Visual Data Exploration and Analysis IV, Proceedings of SPIE 3017, San Jose, Feb. 1997, pp. 206-217.

[3] de Leeuw, W.C., and van Wijk, J.J, Enhanced Spot Noise for Vector Field Visualization, in Proceedings of Visualization '95, pp. 233-239, IEEE Computer Society Press, 1995.

[4] Helman, J.L., and Hesselink, L., Surface Representations of Two- and Three-Dimensional Fluid Flow Topology, in Proceedings of Visualization '90, IEEE Computer Society Press, 1990, pp. 6-13.

[5] Lighthill, M.J., Attachment and Separation in ThreeDimensional Flow, Laminar Boundary Layers, edited by L. Rosenhead II, 2.6:72-82, Oxford University Press, 1963.

[6] Tobak, M., and Peak, D.J., Topology of Three-Dimensional Separated Flows, Annual Review of Fluid Mechanics, Vol. 14., 1982, pp. 61-85.

[7] Wang, K.C., Separation Patterns of a Boundary Layer Over an Inclined Body, AIAA Journal, Vol. 10, No.8, 1972, pp. 1044-1050.

[8] Dallman, U., Topological Structures of Three-Dimensional Vortex Flow Separation, AIAA-83-1935, AIAA 16th Fluid and Plasma Dynamics Conference, 1983.

[9] Zhang, H.X., Chinese Journal of Aerodynamics, No. 4, Dec. 1985, Beijing, Peoples Republic of China.

[10] Chong, M.S., Perry A.E., and Cantwell, B.J., A General Classification of Three-Dimensional Flow Fields, Phys. Fluids A., vol. 2, pp. 765-777, May 1990.

[11] Chong, M.S., and Perry, A.E., Synthesis of two- and threedimensional separation bubbles, 9th Australasian Fluid Mechanics Conference, Auckland, New Zealand, 8-12 December 1986, pp. 35-38.

[12] Chapman, G.T., Topological Classification of Flow Separation on Three-Dimensional Bodies, AIAA-86-0485, AIAA 24th Aerospace Sciences Meeting, Reno, NV, 1986.

[13] Poincare, H., Oeuvres de Henri Poincare, Tome 1, GautheirVillars, Paris, 1928.

[14] Wang, K.C., Zhou, H.C., Hu, C.H., Harrington, S., ThreeDimensional separated flow structure over prolate spheroids, Proc. R. Soc. Lond. A 421, 1990, pp. 73-90.

[15] Brauer, F., and Nohel, J.A., Qualitative Theory of Ordinary Differential Equations, W.A. Benjamin, Inc., 1969, pp. 3395.

[16] Zwillinger, D., Handbook of Differential Equations, 2nd edition, Academic Press, 1957, pp. 360-363.

[17] Ying, S.X., A Numerical Study of Three-Dimensional Separated Flow Past a Hemisphere Cylinder, AIAA-87-1207, AIAA 19th Fluid Dynamics, Plasma Dynamics and Lasers Conference, 1987.

[18] Globus, A., Levit, C., and T.Lasinski, A Tool for Visualizing the Topology of Three-Dimensional Vector Fields, in Proceedings of Visualization '91, IEEE Computer Society Press, 1991, pp. 33-40.

[19] Chaderjian, N.M. and Schiff, L.B., Navier-Stokes Analysis of a Delta Wing in Static and Dynamic Roll, AIAA-95-1868, AIAA Annual CFD Meeting, 1995. 


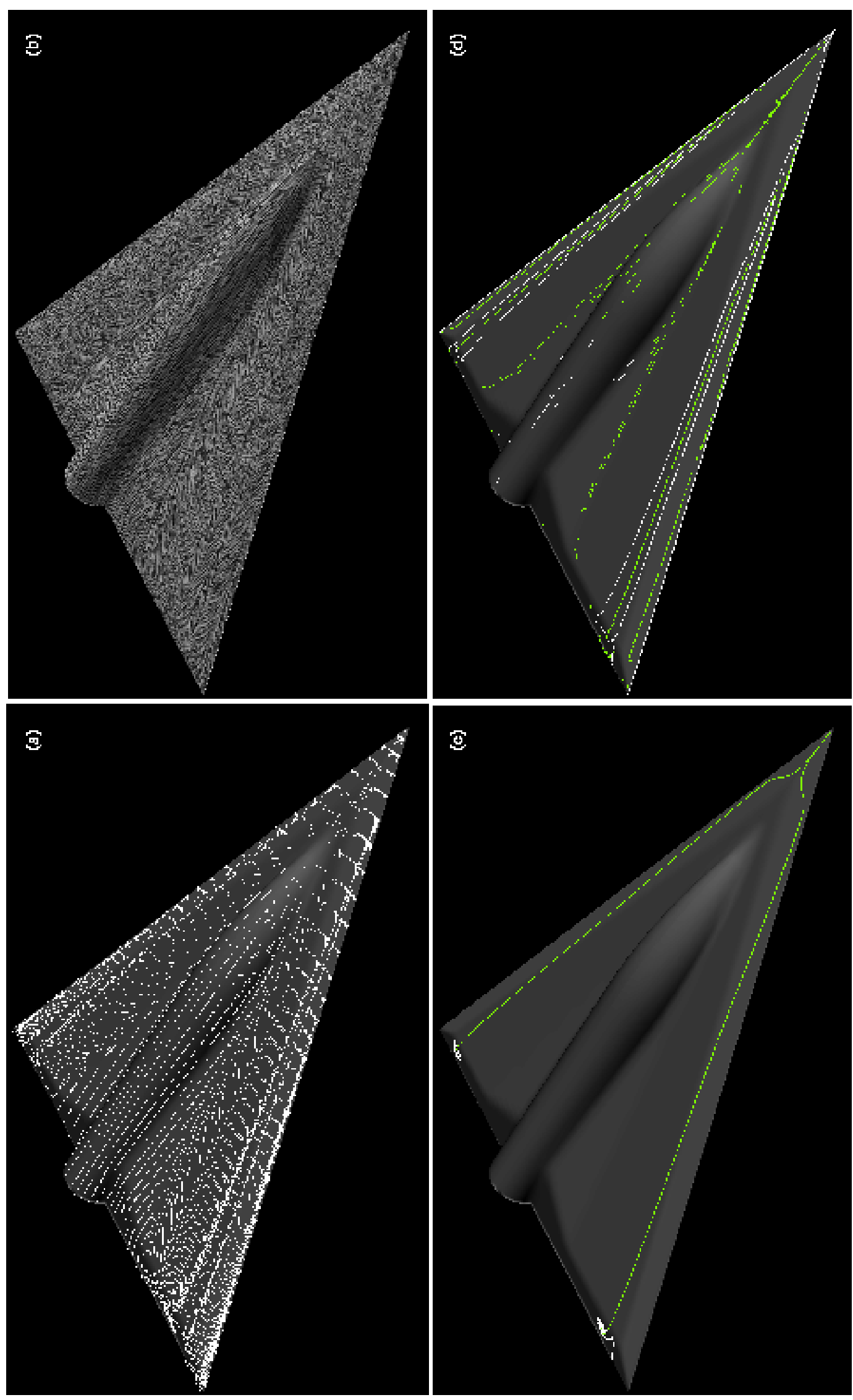

8

窎窎窎 고당 歌察 .ㄷ․ 复哥洝 可 온 무 回要 ᄃ包骂 움뭉 흥듬

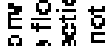
88

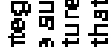
동 달 家它 唯 g 든. 过 的苟骂 $8 \mathrm{E}$ 㗪 可.드몸 或比 ij 옹 $^{\circ}$

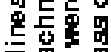
可器 5. E.다 可 苟象要 는도 品琼 ᄃ요 政

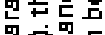
可它要 \% 뭉요 可谓

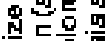
스드응밈 g.joㅇㅎㅇ 哥可器 8 可.드 可品曼 प 먿듬 潘 可吅 듬몯 可 효은 分望

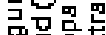
豆员品管 

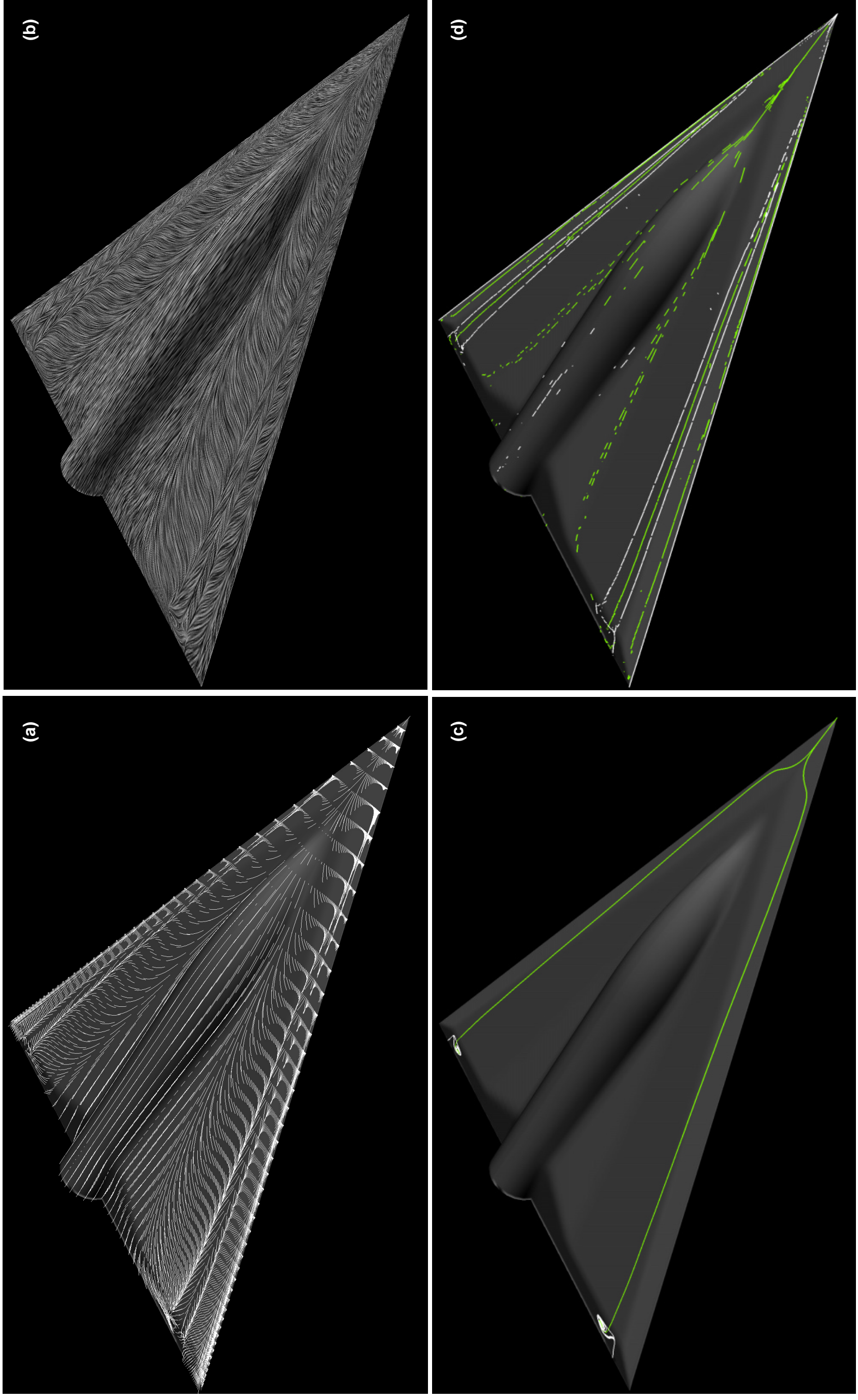

วิ๊

응워

응

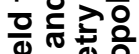

둥워

응흐

응.

㩆

인흥

ฮَ

응

엄르응

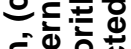

के

음 훙훙

등 흔

츤 흔

o 8

웡

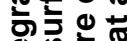

ब的

웡

政

틍으.

可

i.

ब

政.

ब 0 이

¿ 등응

के

Ф

타이응

艛 政.듀 ब舟

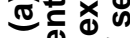

iक

잉

.

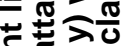

बक

홍

त

.

프응ㅎㅁ

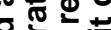

政

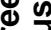

ธ

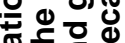

은

응

क्

3 은

은 흐융으

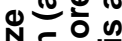

Nㅗ드응

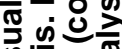

क⿻

우음

का

\&。항

政

중도

ब응

용

용

응응

은

정 응

ㄴㅇㅇ흥

in

흐원워

政

한둥 क 